\title{
Técnicas de Marca de Agua para Video MPEG usando Sensibilidad Visual y Vectores de Movimiento
}

\author{
Antonio Cedillo, Mariko Nakano, Héctor M. Pérez* y Luis M. Rojas \\ Instituto Politécnico Nacional, Sección de Estudios de Posgrado e Investigación, Escuela Superior \\ de Ingeniería Mecánica y Eléctrica Unidad Culhuacán, Av. Santa Ana No. 1000, Col. San Francisco \\ Culhuacán, 04430 Coyoacán, D.F.-México (e-mail: hmpm@prodigy.net.mx)
}

*autor a quien debe ser dirigida la correspondencia

\section{Resumen}

Se propone un algoritmo de marca de agua para video digital, en donde la inserción y detección de la marca de agua se realiza durante el proceso de codificación MPEG-2. La señal de marca de agua esta incrustada en los coeficientes de DCT en el canal azul de los cuadros I y P del video MPEG. La energía de la marca de agua se calcula usando la sensibilidad visual de los cuadros (I y P) y la magnitud de los vectores de movimiento en los cuadros de $\mathrm{P}$. Los resultados de simulación computacional muestran la imperceptibilidad de marca de agua, obteniendo un PSNR mayor a $45 \mathrm{~dB}$, conservando la robustez de la misma cuando es sometida a diversas distorsiones, tales como contaminación por ruido, recorte, y eliminación de cuadros, entre otros. El algoritmo propuesto tiene una influencia mínima en la velocidad del proceso de codificación MPEG.

\section{Video Watermarking Technique using Visual Sensibility and Motion Vector}

\begin{abstract}
A video watermarking algorithm, in which watermark embedding and detection process are carried out during the MPEG-2 coding process is proposed. The watermark signal is embedded in the DCT coefficients of blue channel of I-frames and P-frames. The embedding energy is computed adaptively using perceptual information of the I-frames and the P-frames, and motion vectors information in the P-frame. Computer simulation results show the watermark imperceptibility, obtaining a PSNR greater than $45 \mathrm{~dB}$ and maintaining its robustness to common signal distortions such as contamination by noise, cropping, frame dropping, frame swapping and frame averaging, among others. The proposed algorithm has very little influence on the MPEG decoding speed.
\end{abstract}

Keywords: video watermarking, MPEG-2, DCT coefficients, perceptual sensibility 


\section{INTRODUCCIÓN}

Acompañando al rápido crecimiento del servicio de Internet, el problema del duplicado y alteración ilegal de materiales digitales ha aumentado considerablemente (Langelaar y Lagendijk, 2001). Por lo tanto la protección de derecho de autor sobre materiales digitales es un asunto de suma importancia que requiere una solución inmediata. La técnica de marca de agua se considera como una solución viable para este problema. Hasta la fecha se han propuesto numerosos algoritmos de marca de agua, aunque la gran mayoría de ellos son para imágenes fijas. Generalmente los algoritmos de marca de agua para imágenes fijas no son eficientes cuando se emplean en secuencias de video, debido a que no están consideradas la redundancia temporal de la señal de video ni los ataques comunes a las señales de video (Swanson et al.,1998).

Generalmente en un sistema de marca de agua para la protección de derecho de autor, la marca de agua insertada debe ser imperceptible y robusta contra ataques comunes, tales como: recorte, contaminación por ruido, filtrado y compresión (Wolfgang et al., 1999). Además del requerimiento de imperceptibilidad y robustez, las técnicas de marca de agua para video deben cubrir los siguientes requerimientos: una detección a ciegas, es decir que en el proceso de detección no se requiere la señal de video original, un mecanismo con alta velocidad de proceso para la inserción y detección de la marca de agua y conservación del tamaño del archivo después de la inserción de la marca de agua. Debido a la redundancia existente en las secuencias de video, algunos ataques intencionales que tratan de destruir la marca, tales como la eliminación de algunos cuadros, el intercambio de cuadros y el promedio de cuadros adyacentes, deben considerarse al diseñar algoritmos de marca de agua para video (Wolfgang et al., 1999).

Los algoritmos de marca de agua para video se pueden clasificar en tres categorías: marcas de agua en banda base (Wolfgang et al., 1999; Hartung y Girod 1998; Swanson et al., 1998; Kong et al., 2006), marcas de agua en secuencias codificadas (Wang et al., 2004; Biswas et al., 2005; Langelaar y Lagendijk 2002) y marcas de agua en el proceso de codificación MPEG (Liu et al., 2004; Zhao et al., 2003; Ueno 2004; Noorkami y Mersereau 2006). En las técnicas de marca de agua en banda base la marca de agua se inserta en cuadros de secuencias de video, aquí se pueden usar algoritmos para imágenes fijas, sin embargo el tiempo requerido para la inserción y detección de marcas de agua es considerablemente alto y después de la compresión, tal como la MPEG, la señal de marca de agua se pierde parcialmente o completamente. En el artículo propuesto por Wolfgang et al. (1999), el concepto de JND (Just Noticeable Difference) es usado para determinar la energía adecuada de la marca de agua en el dominio DCT (Discrete Cosine Transform). El algoritmo propuesto por Hartung y Girod (1998), inserta datos binarios modulados por una secuencia pseudo-aleatoria dentro de las componentes de luminancia de los cuadros de video. Swanson et al., (1998), propusieron un algoritmo basado en la Transformada Wavelet a través del tiempo. Kong et al. (2006), aplican descomposición de valores singulares a los cuadros de video e insertan la marca de agua dentro de los valores singulares.

La técnica de marca de agua en secuencias codificadas inserta una señal de marca de agua dentro de secuencias de bits comprimidas por métodos estándar de compresión de video, tales como MPEG-2, MPEG-4, etc. Generalmente esta técnica consume menor tiempo para la inserción y detección de la marca de agua, sin embargo el número de bits de inserción esta limitado por la tasa de compresión. En el artículo propuesto por Wang et al. (2004), la marca de agua se inserta en los cuadros-I de las secuencias de video usando el concepto JND; mientras que Biswas et al. (2005), insertaron marcas de agua directamente en la trama comprimida MPEG mediante la modificación de los coeficientes de DCT. También en el algoritmo propuesto por Langelaar y Lagendijk (2001), la marca de agua se inserta en los cuadros-I en el dominio DCT.

Los algoritmos de marca de agua que se realizan durante el proceso de codificación MPEG son inherentemente robustos contra ataques de compresión estándar, sin incrementar la tasa de bits/s en las secuencias de video. Liu et al. (2004), propusieron un algoritmo donde la marca de agua se inserta en los vectores de movimiento y usando los vectores con la marca de agua se genera la secuencia de bits de MPEG. Mientras Zhao et al. (2003), propusieron un algoritmo rápido para estimar los vectores de movimiento durante el proceso de compresión en el cual la marca de agua se 
inserta modificando ligeramente el ángulo y la magnitud de los vectores de movimiento. En el algoritmo propuesto por Ueno (2004), los vectores de movimiento se usan para determinar la posición dentro de los coeficientes de DCT de los cuadros-I. Noorkami y Mersereau (2006) estimaron regiones de movimiento, calculando la distribución espacial de movimiento de varios cuadros consecutivos y la mayor cantidad de bits de la marca de agua se inserta en las regiones estáticas para evitar el artefacto causado por la marca de agua.

En este artículo, se propone un algoritmo de marca de agua para video, en el cual la inserción de la marca de agua se realiza durante el proceso de codificación MPEG-2. El algoritmo propuesto usa tres criterios basados en el Sistema Visual Humano (SVH) para insertar una marca de agua robusta, preservando su imperceptibilidad. El primer criterio está basado en la sensibilidad del SVH a diferentes canales de color básico, el segundo criterio está basado en el enmascaramiento de textura y borde tomado de la propuesta de Tong y Venetsanopoulos (1998), y el último criterio está basado en los vectores de movimiento en la secuencias de video. El último criterio se aplica únicamente a cuadros- $\mathrm{P}$, mientras que los otros dos criterios se aplican a los cuadros-I y cuadros- $P$. En el algoritmo propuesto, los cuadros-B no se toman en cuenta en los procesos de inserción /detección de marca de agua para reducir la complejidad computacional para no afectar el tiempo de codificación/decodificación de MPEG. Los resultados obtenidos por simulación computacional muestran la imperceptibilidad de la marca de agua y la robustez de la misma contra esquemas comunes de procesamiento de señales y algunos ataques intencionales a la secuencia de video. La imperceptibilidad de la marca de agua se mide usando el PSNR (Peak Signal Noise Ratio) y una evaluación basada en SVH propuesto por Wang y Bovik (2004).

\section{SISTEMA PROPUESTO}

En esta sección proporciona una descripción detallada de los procesos de inserción y detección de la marca de agua en el algoritmo propuesto para video.

\section{Criterios Basados en el SVH}

En el sistema propuesto se usan los tres criterios mencionados anteriormente para poder insertar una marca de agua imperceptible y robusta dentro de una señal de video. Estos tres criterios están basados en la reducida sensibilidad del SVH al canal del color azul, las regiones con detalle como texturas y la capacidad de seguir una región con alta velocidad de movimiento. Existen tres tipos de conos que reaccionan con los tres colores básicos: Rojo, Verde y Azul. El número de conos sensibles al Azul es 30 veces menor que el número de conos sensible a los otros dos colores (Sayood, 2000). La figura 1 muestra la fracción de luz absorbida por cada uno de tres tipos de conos, aquí R, G, B representa los conos sensibles a color Rojo, Verde y Azul, respectivamente. La figura 1 muestra que el SVH tiene menor sensibilidad al color azul que a los otros dos colores básicos (Rojo y Verde).

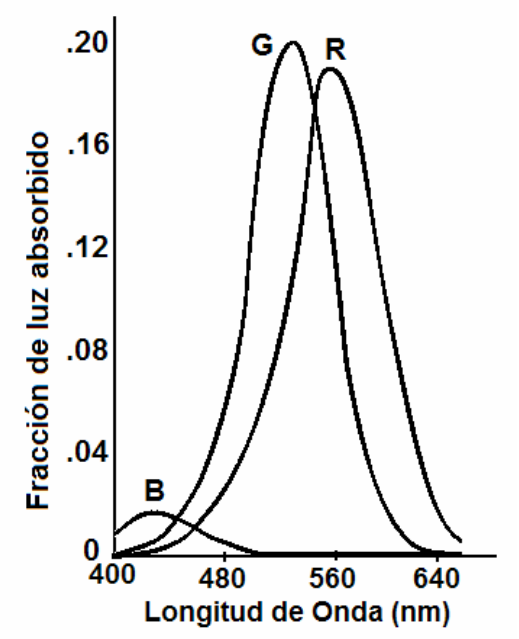

Fig. 1: Sensibilidad de los tres tipos de conos: R(rojo), G(Verde) y B(azul). 
El algoritmo propuesto inserta la marca de agua en el canal Azul, aprovechando la deficiencia del SVH. Para llevar a cabo la inserción, el espacio de color de la señal de video se convierte a RBG, cuando la señal tiene otro espacio de color, tal como YUV o YCrCb, aplicando las matrices de transformación respectivas (Plataniotis y Venetsanopoulos 2000).

El segundo criterio está basado en la diferencia de la sensibilidad del SVH a la región con detalle y a la región plana de una imagen. Cada cuadro-I y cuadro-P es dividido en bloques de 8x8 a los cuales se les aplica la Transformada Coseno Discreta (DCT) Bidimensional. Los bloques se clasifican usando el algoritmo propuesto por Tong y Venetsanopoulos (1998), el cual se describe brevemente a continuación:

1. Cada bloque de la DCT se divide en 4 áreas denotadas por los símbolo DC, L, E y H (figura 2).

2. La suma del valor absoluto de los coeficientes que pertenecen a las 4 áreas se calcula y se denota como $S_{D C}, S_{L}, S_{E}$ y $S_{H}$, respectivamente.

3. Usando las siguientes condiciones, cada bloque de la DCT se clasifica como "bloque de borde", "bloque de textura" o "bloque plano".

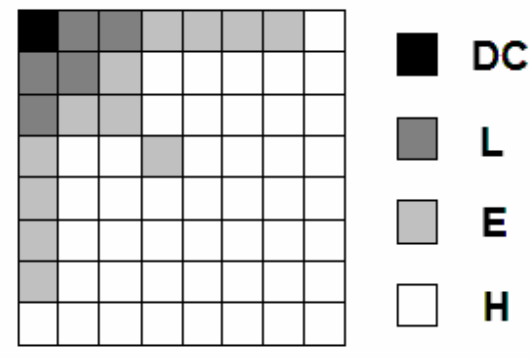

Fig. 2: Cuatro áreas de bloque DCT

Condiciones para el bloque de borde

Si alguna de las siguientes condiciones (condición-A o condición-B) se satisface, entonces el bloque de la DCT se clasifica como "bloque de borde".

\section{Condición-A:}

$\operatorname{Si~}\left(S_{E}+S_{H} \leq \mu_{2}\right) \wedge\left(\frac{S_{L}}{S_{E}} \geq \alpha_{1}\right) \wedge\left(\frac{S_{L}+S_{E}}{S_{H}} \geq \beta_{1}\right) \quad$ o

$\operatorname{Si}\left(S_{E}+S_{H} \leq \mu_{2}\right) \wedge\left(\frac{S_{L}}{S_{E}} \geq \beta_{1}\right) \wedge\left(\frac{S_{L}+S_{E}}{S_{H}} \geq \alpha_{1}\right)$ o

$\operatorname{Si}\left(S_{E}+S_{H} \leq \mu_{2}\right) \wedge\left(\frac{S_{L}+S_{E}}{S_{H}} \geq \gamma\right)$
Condición-B

Si $\left(S_{E}+S_{H}>\mu_{2}\right) \wedge\left(\frac{S_{L}}{S_{E}} \geq \alpha_{2}\right) \wedge\left(\frac{S_{L}+S_{E}}{S_{H}} \geq \beta_{2}\right)$ o

$\operatorname{Si~}\left(S_{E}+S_{H}>\mu_{2}\right) \wedge\left(\frac{S_{L}}{S_{E}} \geq \beta_{2}\right) \wedge\left(\frac{S_{L}+S_{E}}{S_{H}} \geq \alpha_{2}\right)$ o

Si $\left(S_{E}+S_{H}>\mu_{2}\right) \wedge\left(\frac{S_{L}+S_{E}}{S_{H}} \geq \gamma\right)$

donde el operador $\wedge$ significa multiplicación lógica siendo los valores de los seis parámetros son determinados experimentalmente: $\mu_{2}=900$,

$\alpha_{1}=2.3, \beta_{1}=1.6, \alpha_{2}=1.4, \beta_{2}=1.1$ y $\gamma=4$.

Condición para el bloque de textura

Si la Condición-A no se satisface y $S_{E}+S_{H}>\kappa$; o la condición-B no se satisface entonces el bloque de la DCT se clasifica como "bloque de textura", donde $\kappa=290$.

\section{Condición para el bloque plano}

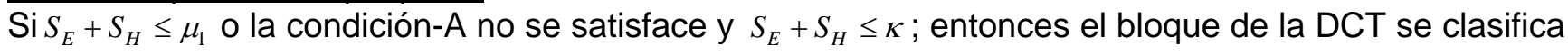
como "bloque plano", donde $\mu_{1}=125$. 


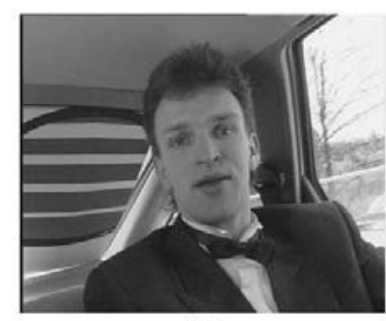

(a)

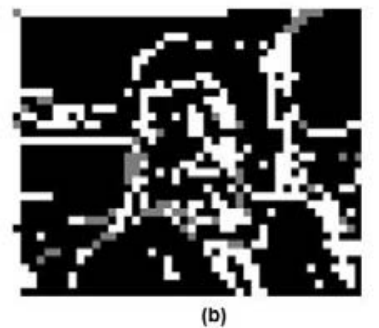

(b)

Fig. 3: Ejemplo de la clasificación de bloques usando el modelo visual.

La figura 3 muestra un ejemplo de clasificación de bloques usando el algoritmo de Tong y Venetsanopoulos (1998), descrito anteriormente. Aquí los bloques negros son bloques planos, los bloques grises son bloques de textura y los bloques blancos son bloques de borde, respectivamente.

El último criterio está basado en la deficiencia del SVH para seguir regiones con movimiento de alta velocidad. La predicción de compensación de movimiento es una poderosa herramienta para reducir la redundancia temporal en la codificación de MPEG. Los macro-bloques con mayor magnitud del vector de movimiento se pueden clasificar como regiones de movimiento con alta velocidad, en los cuales se puede insertar una marca de agua con mayor fuerza sin que cause una degradación en la señal de video. La magnitud del vector de movimiento se calcula usando la ecuación (1).

$M m v_{i}=\sqrt{m v h_{i}^{2}+m v v_{i}^{2}}, \quad i=1 . . M B$

donde $m v h_{i}, m v v_{i}$ son las componentes horizontal y vertical del vector de movimiento del i-ésimo macro-bloque y $M B$ es el número total de macro-bloques. Para determinar la región con un movimiento de alta velocidad, se introduce un valor de umbral Th_mv y cada macro-bloque se clasifica como bloque con movimiento de alta velocidad y bloque con movimiento de baja velocidad o sin movimiento en la siguiente forma:

Si $M m v_{i}<T h \_m v$ entonces bloque es estático

Si $M m v_{i} \geq T h_{-} m v$ entonces bloque es de movimiento

donde el valor del umbral Th_mv se calcula por medio de la ecuación (2).

$T h \_m v=\frac{1}{M B} \sum_{i=1}^{M B} M m v_{i}$

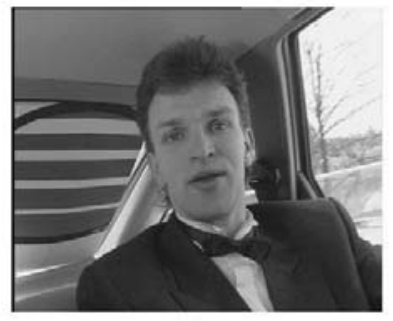

(a)

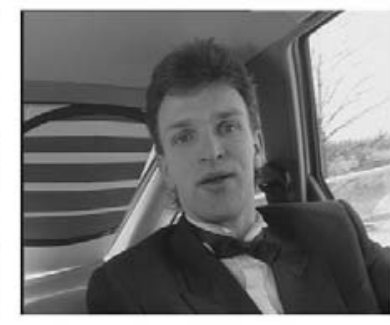

(b)

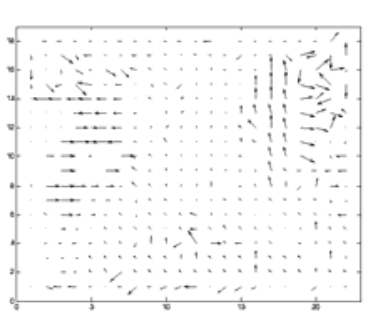

(c)

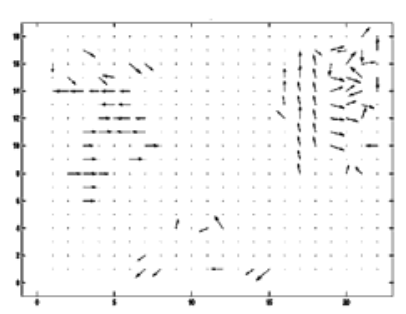

(d)

Fig. 4: Dos cuadros consecutivos de video y los vectores de movimiento antes y después de clasificación.

La figura 4 muestra dos cuadros consecutivos de video (fig. 4(a) y (b)) y los vectores de movimiento antes de la clasificación (fig. 4(c)) y después de la clasificación (fig. 4(d)). 
Combinando los dos últimos criterios: las características de los bloques de tamaño 8x8 y la velocidad de movimiento del macro-bloque (16×16), la energía de inserción de la marca de agua para cuadros-I y cuadros- $P$ es determinada experimentalmente usando 10 secuencias de videos. Los valores de energía se muestran en la tabla 1 . En la tabla, $B$ es el bloque de $8 \times 8$ píxeles en cuadros-I o cuadros$\mathrm{P}$ y $M b_{\text {motion }}$ es el macro-bloque con movimiento de alta velocidad, que contiene 4 bloques $B$. $C_{I}, C_{P}$ son cuadros-I y cuadros-P, respectivamente. La figura 5 muestra un ejemplo de la clasificación de bloques junto con la energía de inserción de la marca de agua asignada a cada bloque.

Tabla 1: Energía de inserción de la marca de agua

\begin{tabular}{|l|c|c|c|}
\hline Energía & $B \in$ & $B \in$ & $B \in$ \\
& Plano & Textura & Borde \\
\hline
\end{tabular}

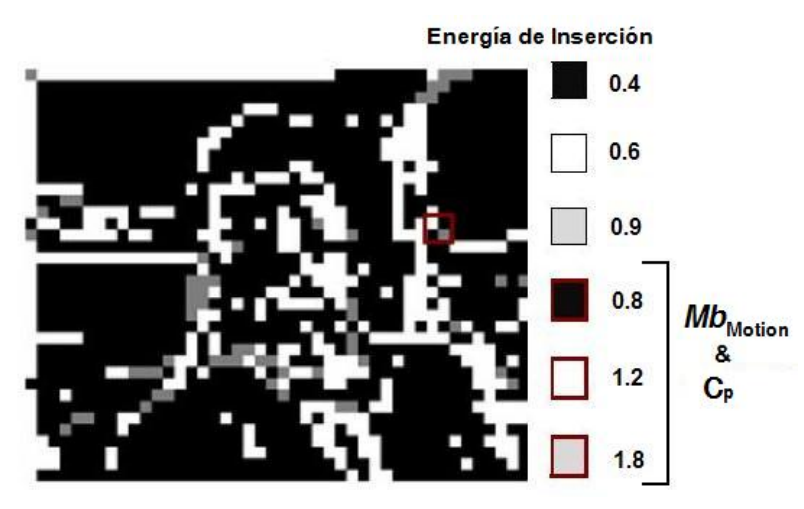

Fig. 5: Un ejemplo de asignación de la energía de inserción de la marca de agua a cada bloque.

\section{Proceso de Inserción de la Marca de Agua}

El proceso de inserción de marca de agua en el algoritmo propuesto consiste de dos partes: la primera parte es el cálculo de energía de inserción de la marca de agua mencionado en la sección anterior. La segunda parte es la inserción de la marca de agua en cada bloque usando la energía calculada en la primera parte. Primero en el espacio de color RBG se aísla el canal azul al cual se le aplica la DCT cuyos coeficientes se dividen en bloques de 8x8 píxeles, seguidamente cada bloque se clasifica en una de las tres categorías (bloque plano, bloque de textura y bloque de borde). Para los cuadros-P los macro-bloques (16x16 píxeles) se clasifican en bloque con movimiento o bloque sin movimiento. Después de las clasificaciones tanto en el bloque de 8x8 como en los macro-bloques de 16×16, se realiza la asignación de energía de la inserción de la marca de agua. La señal de marca de agua es una secuencia pseudo-aleatoria generada por la llave secreta. La inserción de la marca de agua se realiza por medio de la ecuación (3).

$$
\begin{aligned}
& D_{C T}(i, j)=D_{C} T_{k}(i, j)+\alpha_{k}\left|D C T_{k}(i, j)\right| W_{k} \\
& (i, j)=(1,2) \text { para } C_{\mathrm{I}} \\
& (i, j) \in\{(1,2),(1,3),(2,1),(2,2),(3,1)\} \text { para } C_{\mathrm{P}}
\end{aligned}
$$

donde $D C T_{k}$ denota el k-ésimo bloque de la DCT y $\alpha_{k}$ es la energía de inserción del bloque-k. Para los cuadros-I, la marca de agua se inserta en un coeficiente de AC con la frecuencia más baja y para los cuadros-P, la marca de agua se inserta en los 5 coeficientes AC con las frecuencias más bajas. La figura 6 muestra el esquema del proceso de inserción. 


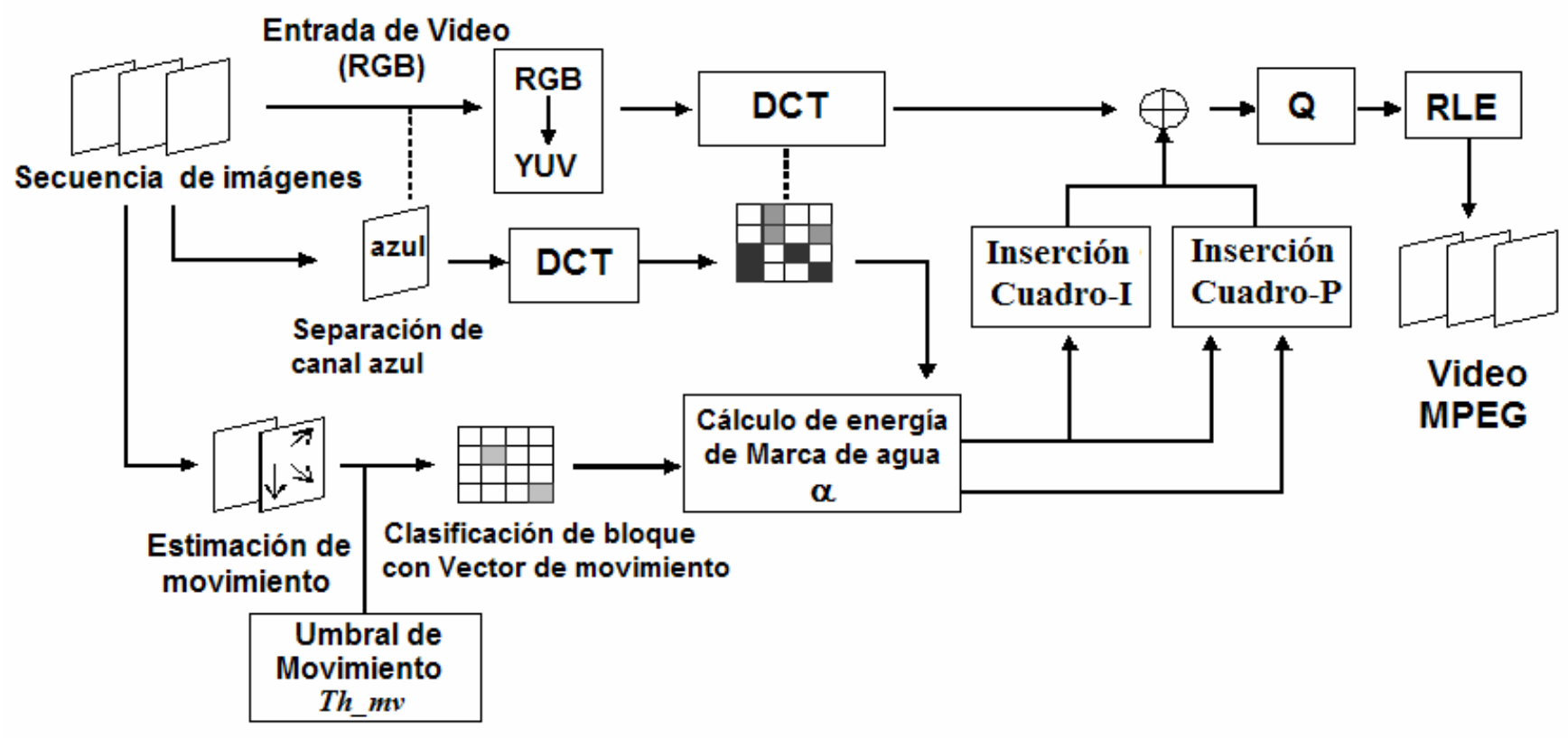

Fig. 6: Proceso de inserción

\section{Proceso de Detección de la Marca de Agua}

En el proceso de detección de la marca de agua, la aplicación de la DCT, la determinación de la energía de la marca en cada bloque son iguales a los procesos realizados en la inserción. Los coeficientes marcados son formados el primero y segundo coeficiente de cada bloque de la DCT del cuadro-I marcado y los 5 primeros coeficientes de cada bloque la DCT del cuadro-P marcado. La correlación cruzada entre los coeficientes marcados y la marca de agua del propietario se calcula como se muestra en la ecuación (4).

$C=\frac{1}{L} \sum_{i=1}^{L} W_{i} Y_{i}$

donde $W$ es la marca de agua con la que el propietario esta reclamando su propiedad, $Y$ es el vector de coeficientes de la DCT extraídos de la secuencia de video marcado y probablemente distorsionado, mientras que $L$ es la longitud de la marca de agua.

La marca de agua del dueño es detectada, si el valor de la correlación es mayor que un valor del umbral determinado, el cual se muestra en la ecuación (5). Este valor determina la probabilidad del error de detección y la probabilidad de error de falsa alarma. En el algoritmo propuesto se usó un valor adaptable del umbral que garantiza que el error de falsa alarma sea menor a $10^{-6}$ (Piva et al., 1997).

$T h_{w}=\frac{1}{3 L} \sum_{i=1}^{L}\left|Y_{i}\right| \alpha_{i}$

\section{RESULTADOS EXPERIMENTALES}

Para evaluar el algoritmo propuesto, usamos varias secuencias de video con formato YUV-CIF. La figura 7 muestra algunas de las secuencias de video usadas para la evaluación. 


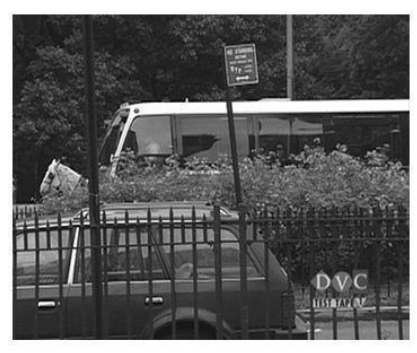

(a)

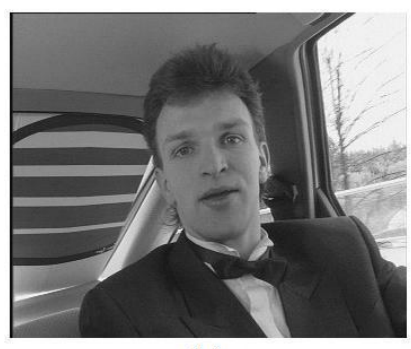

(b)

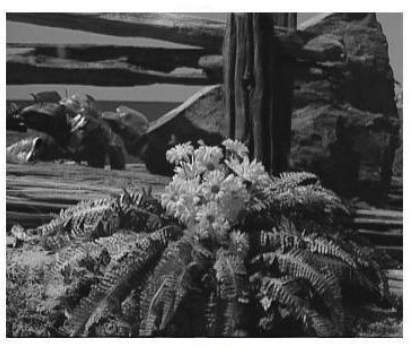

(c)

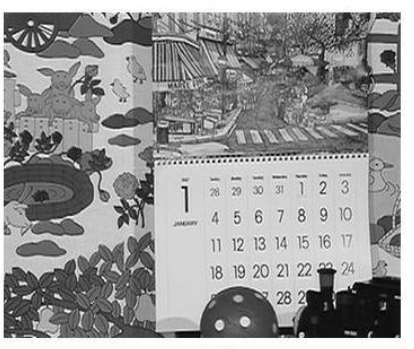

(d)

Fig. 7: Algunas secuencias de video usadas para la evaluación del sistema.

\begin{tabular}{|c|c|c|c|c|c|}
\hline Cuadro Original & Cuadro Marcado & $\begin{array}{c}\text { PSNR } \\
\text { (dB) }\end{array}$ & Cuadro Original & Cuadro Marcado & $\begin{array}{c}\text { PSNR } \\
\text { (dB) }\end{array}$ \\
\hline Cuadro-I & Cuadro-I & & Cuadro-P & \\
\hline
\end{tabular}

Fig. 8: Imperceptibilidad de la marca de agua en el cuadro-I y el cuadro-P

El algoritmo propuesto es evaluado desde el punto de vista de imperceptibilidad y robustez de la marca de agua insertada.

\section{Imperceptibilidad}

La imperceptibilidad de la marca de agua insertada en el algoritmo propuesto es evaluada usando el PSNR y el índice de calidad universal propuesto por Wang y Bovik (2004), el cual realiza una medición objetiva relacionada con la distorsión percibida por el SVH. El rango de los valores del índice es [0.0 1.0], cuando la secuencia de video bajo análisis es idéntica numéricamente a la original, el valor del índice es igual a 1.0. La figura 8 muestra el cuadro-I original, el cuadro-I marcado, el cuadro-P original y el cuadro-P marcado, junto con el respectivo valor de PSNR. El índice de calidad universal de la secuencia de video marcada respecto al original es aproximadamente igual a 0.96.

Como se puede observar de la figura, para cada cuadro de video, la marca de agua insertada es imperceptible, ya que el valor del PSNR es alrededor de 40dB en ambos tipos de cuadro. El valor del índice universal de calidad de video nos indica que la marca de agua es totalmente imperceptible para el SVH, cuando se evalúa como secuencia de vídeo.

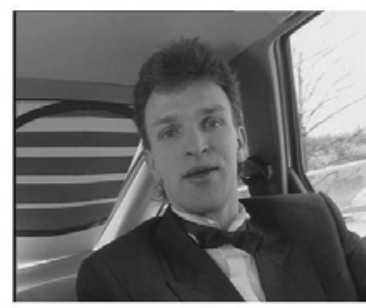

(a)

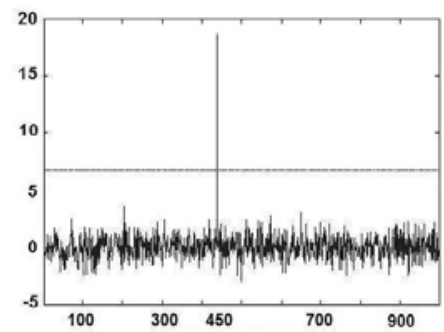

(b)

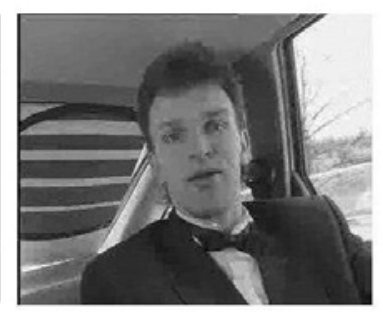

(c)

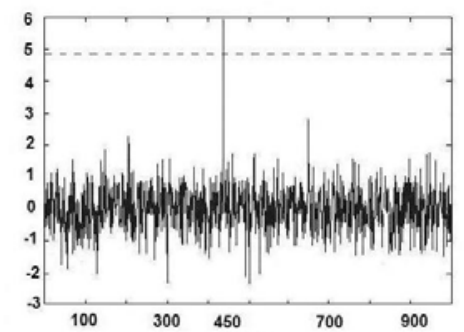

(d)

Fig. 9: (a) cuadro marcado, (c) cuadro marcado y comprimido, (b) y (d) sus respuestas del detector. Robustez de la Marca de Agua 
Para evaluar la robustez de la marca de agua en el algoritmo propuesto, las secuencias de video marcadas son atacadas usando algunos procesamientos comunes de señales e imágenes, tales como el cambio de tasa de codificación, contaminación por ruido impulsivo y ruido Gaussiano, la eliminación de cuadros, el Intercambio de cuadros, promedio de cuadros y el recorte. La figura 9 muestra la robustez de la marca de agua contra el cambio de la tasa de codificación, aplicando diferentes matrices de cuantificación en la codificación de entropía en el proceso de MPEG. La figura 9 muestra: (a) un cuadro de video marcado sin ataque, (c) un cuadro de video marcado y comprimido con el factor de calidad de 30, (b) y (d) las respuestas del detector cuando éste analiza el video marcado (a) y (c) con 1000 posibles marcas de agua diferentes. De esta figura podemos concluir que la marca de agua insertada es robusta a altas tasas de compresión. La marca de agua sobrevive aún cuando se aplique una tasa de compresión con un factor de calidad tan bajo como 30. Cuando se aplica un factor de calidad menor a 30, la marca de agua se podría perder, aunque en esta situación, la calidad de video es demasiado pobre y el video pierde su valor comercial.

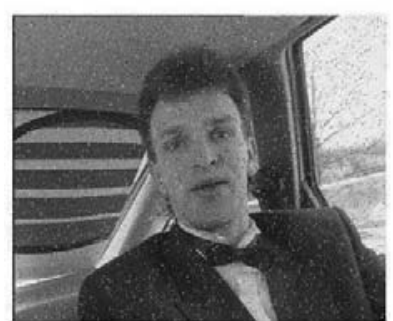

(a)

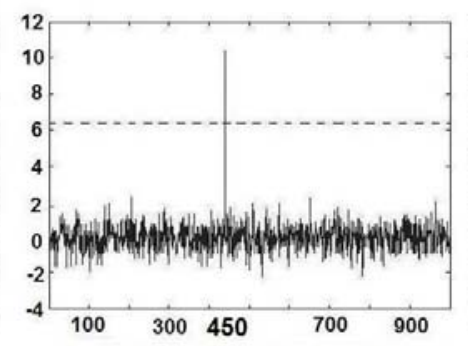

(llave de dueño) (b)

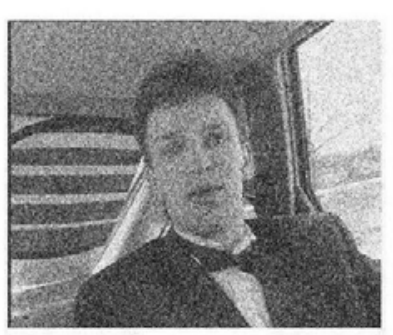

(e)

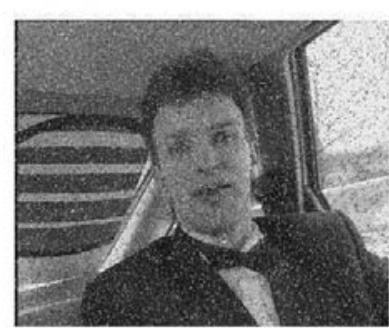

(c)

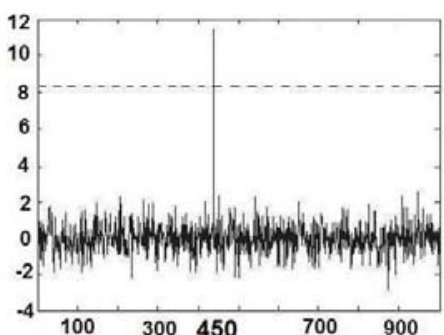

(llave de dueño) (d)

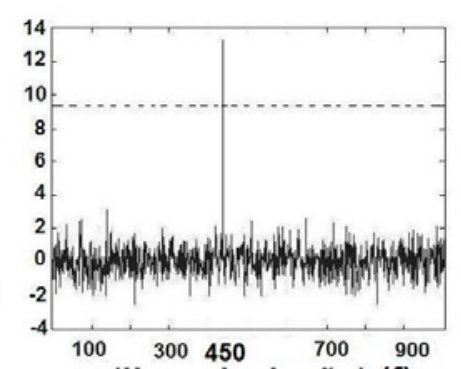

(llave de dueño) (f)

Fig. 10: Robustez de marca de agua contra contaminación de ruido.

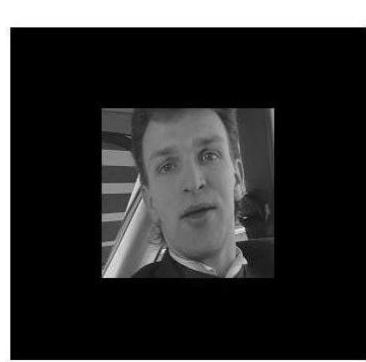

(a)

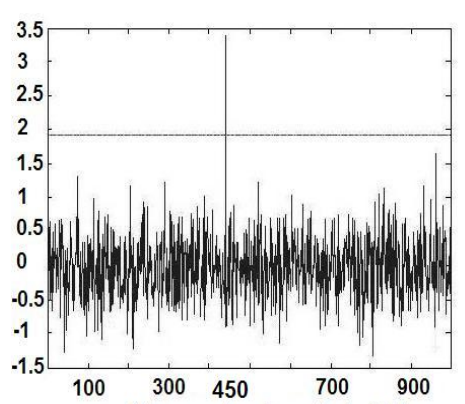

(llave de dueño) (b)

Fig. 11: Robustez de la marca de agua contra recorte. (a) $75 \%$ de datos recortado de cada cuadro de video marcado, (b) respuesta de detector.

La figura 10 muestra la robustez de la marca de agua contra la contaminación por ruido impulsivo y Gaussiano. La figura 10 muestra: (a) y (c) los cuadros marcados y contaminados por ruido impulsivo con densidad de 3\%, 10\%, respectivamente, (b) y (d) las respuestas del detector, (e) un cuadro marcado y contaminado por ruido Gaussiano con varianza de 0.05 y (f) la respuesta del detector. De la figura podemos concluir que la marca de agua es suficientemente robusta contra este tipo de ataques. También en el algoritmo propuesto, la marca de agua es robusta contra recorte, la figura 11 muestra el funcionamiento de detección de la marca de agua cuando $75 \%$ de cada cuadro de la secuencia de video marcada es eliminado. En las figuras 8-10, se muestran los valores de la 
correlación cruzada calculada en la ecuación (4), aplicando 1000 marcas de agua generadas con diferentes llaves (1-1000), junto con valor umbral calculado en la ecuación (5). En todos los casos la llave secreta del dueño es la llave 450.

Eliminación de cuadros, intercambio de cuadros y promedio de cuadros son ataques intencionales para una secuencia de video (Zhuang et al., 2004). Estos ataques aprovechan redundancia temporal de la secuencia de video y tratan de eliminar la marca de agua insertada sin causar una degradación visual apreciable de la señal de video. Estos ataques se describen en las ecuaciones (6), (7) y (8).

\section{Eliminación de cuadros}

$V_{\text {atacado }}=V_{\text {original }}-\left\{C_{r 1}, C_{r 2}, \ldots \ldots C_{r n}\right\}$

Intercambio de cuadros

$\tilde{C}_{k} \Leftrightarrow C_{k+1} \tilde{C}_{k+1} \Leftrightarrow C_{k}$

Promedio de cuadros

$\tilde{C}_{k}=\frac{1}{3}\left[C_{k-1}+C_{k}+C_{k+1}\right]$

donde $V_{\text {atacado }}, V_{\text {marcado }}$ son secuencias de video modificadas por el ataque y marcado, respectivamente, $C_{k}, \tilde{C}_{k}$ son el k-ésimo cuadro de video marcado y atacado. [r1, $\left.r 2, \ldots . . r n\right]$ es el número correspondiente a los cuadros seleccionados de una manera aleatoria.

Debido a que el algoritmo propuesto inserta la marca de agua a lo largo de la secuencia temporal de video, la marca de agua insertada es inherentemente robusta contra estos ataques.

\section{Velocidad de inserción y detección}

En las técnicas de marca de agua para video, el consumo de tiempo ocasionado por la inserción y detección de la marca de agua debe ser el mínimo posible dentro de la codificación MPEG. Por lo tanto se realizó una medición del consumo de tiempo empleado en la codificación MPEG sin y con inserción/detección de marca de agua. Aquí el consumo de tiempo aumentó menos del 10\% del tiempo requerido para la codificación MPEG. Esto significa que el algoritmo propuesto es factible para una implementación real.

\section{Comparación con Otros Algoritmos}

El algoritmo propuesto se comparó con otros algoritmos previamente propuestos para el mismo objetivo: los algoritmos que se usaron para la comparación son los siguientes: a) Algoritmo de inserción y detección de marca de agua en banda base usando Transformada Wavelets 3D (Zhuang et al., 2004). b) El algoritmo basado en la modificación de vector de movimiento realizado durante el proceso de codificación MPEG (Zhao et al., 2003). c) El algoritmo basado en el dominio de DCT donde la inserción se realiza durante el proceso de codificación MPEG (Zhang et al., 2001).

La tabla 2 muestra una comparación de robustez de la marca de agua insertada, aquí 'O' significa que la marca de agua es robusta contra el ataque indicado y ' $X$ ' significa que la marca de agua se pierde después del ataque. Como se puede observar de la tabla 2, el algoritmo propuesto ofrece mayor robustez de la marca de agua, comparado con otros algoritmos. 
Tabla 2: Comparación entre tres algoritmos propuestos por otros autores y el algoritmo propuesto.

\begin{tabular}{|l|c|c|c|c|}
\hline Ataques & A & B & C & Propuesto \\
\hline MPEG & $\mathbf{X}$ & $\mathbf{O}$ & $\mathbf{O}$ & $\mathbf{O}$ \\
\hline $\begin{array}{l}\text { Promedio } \\
\text { de cuadros }\end{array}$ & $\mathbf{O}$ & $\mathbf{O}$ & $\mathbf{X}$ & $\mathbf{O}$ \\
\hline $\begin{array}{l}\text { Eliminación } \\
\text { de cuadros }\end{array}$ & $\mathbf{O}$ & $\mathbf{O}$ & $\mathbf{O}$ & $\mathbf{O}$ \\
\hline $\begin{array}{l}\text { Intercambio } \\
\text { de cuadros }\end{array}$ & $\mathbf{O}$ & $\mathbf{O}$ & $\mathbf{O}$ & $\mathbf{O}$ \\
\hline Recorte & $\mathbf{X}$ & $\mathbf{X}$ & $\mathbf{O}$ & $\mathbf{O}$ \\
\hline $\begin{array}{l}\text { Ruido } \\
\text { impulsivo. }\end{array}$ & $\mathbf{O}$ & $\mathbf{X}$ & $\mathbf{O}$ & $\mathbf{O}$ \\
\hline $\begin{array}{l}\text { Ruido } \\
\text { Gaussiano }\end{array}$ & $\mathbf{O}$ & $\mathbf{X}$ & $\mathbf{O}$ & $\mathbf{O}$ \\
\hline
\end{tabular}

\section{CONCLUSIONES}

El algoritmo propuesto realiza la inserción y detección de la marca de agua durante la codificación de MPEG-2, obteniendo la energía adecuada para la inserción de la marca de agua a partir de la información visual de los cuadros I y $\mathrm{P}$ en el dominio DCT y del valor de los vectores de movimiento.

El algoritmo propuesto fue evaluado desde el punto de vista de imperceptibilidad y robustez de la marca de agua insertada. La imperceptibilidad de la marca de agua se evaluó usando el PSNR y el índice de calidad visual. Ambos valores muestran la imperceptibilidad de la marca, sobre todo la marca de agua es invisible cuando se observa como secuencia de video. Para evaluar robustez de la marca de agua, algunos ataques comunes incluyendo ataques para video fueron introducidos. Los resultados de simulación y la comparación con otros algoritmos para el mismo objetivo muestran que el algoritmo propuesto es superior a otros previamente propuestos y ofrece suficiente robustez contra una importante gama de posibles ataques.

A partir de la medición del consumo de tiempo agregado al requerido por la codificación de MPEG, se puede concluir que el uso del algoritmo propuesto es factible para una implementación en problemas reales de protección de derechos de autor.

\section{AGRADECIMIENTOS}

Al Consejo Nacional de Ciencia y Tecnología (CONACyT) por el apoyo dado para la realización de este trabajo.

\section{REFERENCIAS}

Biswas S., S.R. Das y E.M. Petriu; An Adaptive Compressed MPEG-2 Video Watermarking Scheme, IEEE Trans. on Instrumentation and Measurement: 54(5), 1853-1861 (2005).

Hartung, F. y B. Girod; Watermarking of uncompressed and compressed video, Signal Processing: 66, 283-301 (1998)

Kong W., B. Yang, D. Wu y X. Niu; SVD Based Blind Video Watermarking Algorithm, IEEE Int. Conf. on Innovative Computing, Information and Control: 1, 265-268 (2006)

Langelaar, G.C. y R.L. Lagendijk; Optimal differential energy watermarking of DCT encoded images and video, IEEE Trans. on Image Processing: 10(1), 148-158 (2001). 
Liu, Z., H. Liang, X. Niu y Y. Yang; A Robust Video Watermarking in Motion Vectors, IEEE Int. Conf. Signal Processing: 2358-2361 (2004).

Noorkami M. y R.M. Mersereau; Improving Perceptual Quality in Video Watermarking Using Motion Estimation, IEEE Int. Conf. on Image Processing (ICIP): 2, 520-523 (2006).

Piva, A., M. Barni, F. Bartolini y V. Cappellini; DCT-Based Watermark Recovering without Resorting to the Uncorrupted Original Image, IEEE Int. Conf. on Image Processing (ICIP): 1, 520-523 (1997).

Plataniotis, N y A.N. Venetsanopoulos; Color Image Processing and Application, First Edition, Springer-Verlag (2000).

Sayood K.; Introduction to Data Compression, $2^{\text {nd }}$ Edition, Morgan Kaufmann Publishers (2000).

Swanson, M.D., B. Zhu y A.H. Tewfik; Multi-resolution scene-based video watermarking using perceptual models, IEEE J. Select areas Communication: 16, 540-550 (1998).

Tong, H.Y. y A.N. Venetsanopoulos; A Perceptual model for JPEG applications based on block classification, texture masking and luminance masking, Int. Conf. on Image Processing (ICIP): 3, 428432 (1998).

Ueno, Y.; A Digital Video watermarking method by association with the Motion Estimation, IEEE Int. Conf. on Signal Processing (ICSP), 2576-2579 (2004).

Wang, J., A.R. Steele y J. Liu; Efficient Integration of Watermarking with MPEG Compression, IEEE Int. Conf. on Multimedia and Expo (ICME): 911-914 ( 2004)

Wang, Z., Lu L. y Bovik A. C.; Video quality assessment based on structural distortion measurement, Elsevier Signal Processing: Image Communication: 19, 121-132 (2004).

Wolfgang, R.B, C.I. Podilchuk y E.J. Delp; Perceptual watermarks for digital images and video, Proceeding IEEE: 87(7), 1108-1126 (1999).

Zhao, Z., N. Yu y X. Li; A Novel Video Watermarking Scheme in Compressed Domain Based of Fast Motion Estimation, IEEE Int. Conf. on Communication Technology (ICCT), 1878-1882 (2003)

Zhang, J., J. Li, y L. Zhang; Video Watermark Technique in Motion Vector, XIV Brazilian Symposium on Computer Graphics and Image Processing: 179-182 (2001).

Zhuang, H., Y. Li y C. Wu; A Blind Spatial-temporal Algorithm based on 3D Wavelet for Video Watermarking, IEEE int. Conf. on Multimedia and Expo (ICME): 3, 1727-1730 (2004). 